\title{
An Equivalent Circuit Approach for Microstrip Component Analysis Using the FDTD Method
}

\author{
L. L. Liou, Member, IEEE, M. Mah, Member, IEEE, and J. Cook
}

\begin{abstract}
The conventional analysis of waveguide parameters for microstrip components using the finite-difference timedomain (FDTD) method is based on monitoring the traveling wave at multiple locations. The dispersion relations of the effective dielectric constant and the characteristic impedance usually exhibit oscillatory behavior. An equivalent circuit approach was utilized for these parameters. Together with the FDTD results, the inductance and the capacitance were evaluated. The phase velocity and the characteristics impedance were then calculated at a single monitor location. With this method, the oscillatory features in the dispersion relations were minimized.
\end{abstract}

Index Terms - Characteristic impedance, FDTD, microstrip, microwave circuit, phase velocity.

\section{INTRODUCTION}

$\mathbf{T}$ HE finite-difference time-domain (FDTD) method is a popular technique to solve electromagnetic propagation problems in numerous areas, including planar circuitry using monolithic microwave integrated circuit (MMIC) technology [1]-[8]. The method is popular since Maxwell's equations can be discretized in a straightforward manner. In addition, the solutions resulting from FDTD provide useful information in the time domain, which is complementary to the frequency domain information.

The microstrip line is the backbone of the waveguiding device in MMIC's. Numerous analytical and numerical methods, including FDTD, have been performed to solve for its waveguiding properties. In FDTD analysis, the frequency dependence (dispersion relation) of the microwave parameters of the wave characteristic impedance and the effective dielectric constant show oscillatory behavior. This behavior is not seen, however, in the results using full-wave frequencydomain analysis. This behavior may be due to the following reasons: 1) imperfect absorbing boundary conditions (ABC's) that generate artificial reflection waves at the computational boundary; 2) the excitation source can contain mixed modes of propagation and evanescent waves; 3 ) the continuous radiation loss spectrum that was taken into account in FDTD, but failed to be implemented properly in the multiple- $z$ method (see below) for the phase velocity determination; and 4) possible numerical errors due to the numerical approximation of the Fourier integration. Among these reasons, the first one due to the imperfect absorption boundary condition is believed to be the main cause of the oscillatory behavior. Several methods

Manuscript received May 22, 1998.

The authors are with Sensor Directorate, Air Force Research Laboratory, Wright-Patterson AFB, OH 45433-7322 USA.

Publisher Item Identifier S 1051-8207(98)08533-X. have been proposed to remove this anomalous behavior. One method is to introduce artificial boundary conditions, such as an open and a short to obtain the correct average value [3]. Other methods have applied an improved ABC such as a dispersive boundary condition [4] or even a higher order of the general ABC [5]. Some have applied signal processing techniques, such as Prony's spectral domain method, to reduce the error due to the residual reflection from the imperfect $A B C$ [6], [7]. In these approaches, multiple observation locations in the propagation direction were required to monitor the wave propagation at different frequencies. Comparing the phase for each frequency component at these locations, the propagation constant can be derived with the knowledge of the wavetraveling distance between these monitoring locations. This method of phase velocity determination is called the multiple- $z$ method.

The multiple- $z$ method relies on the phase difference between different time-series signals at different $z$-locations in the propagation direction. The error in the derived phase velocity due to imperfections in each of the time-series signals is qualitatively additive. This method is, thus, prone to the effects described above. In addition, the multiple of an integer with $2 \pi$ in the phase difference creates a nuisance when trying to determine a unique propagation constant. In this letter, we propose an equivalent circuit approach to solve the oscillatory problem. This approach needs only one observation point in the propagation direction to monitor the wave and to calculate the dispersion relation of the phase velocity; it is, therefore, also called the single- $z$ method. We will show that the microwave parameters, including phase velocity and characteristic impedance derived using this approach, are less sensitive to the adverse effects and the oscillatory behavior is minimized. In Section II, a brief theory of the equivalent circuit approach is given. In Section III, the numerical results and discussion are presented, followed by the conclusion.

\section{EQuivalent Circuit APPROACH}

In FDTD analysis, the time-dependent electric and magnetic field distributions are computed at interlaced locations forming Yee's lattice with a leapfrog time marching scheme [8]. The equivalent circuit components of the inductance per unit length $L$ and the capacitance per unit length $C$ can be derived from the computed $E$ - and $H$-field distributions. They are calculated using the following equations:

$$
\begin{aligned}
& L \delta z=\psi / I \\
& C \delta z=Q / V
\end{aligned}
$$




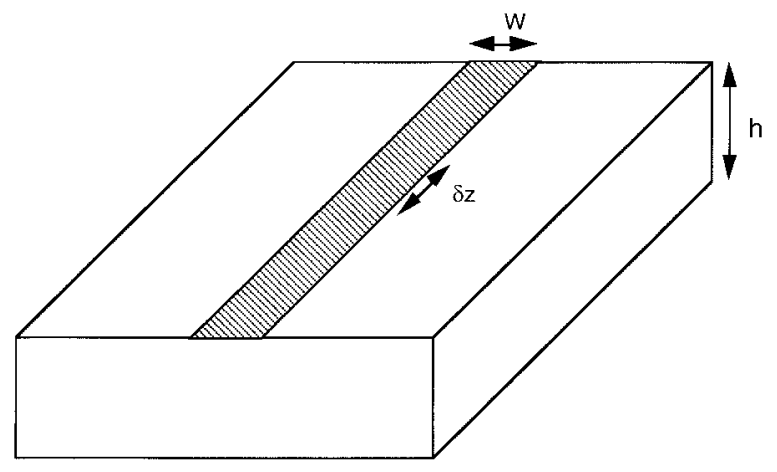

(a)

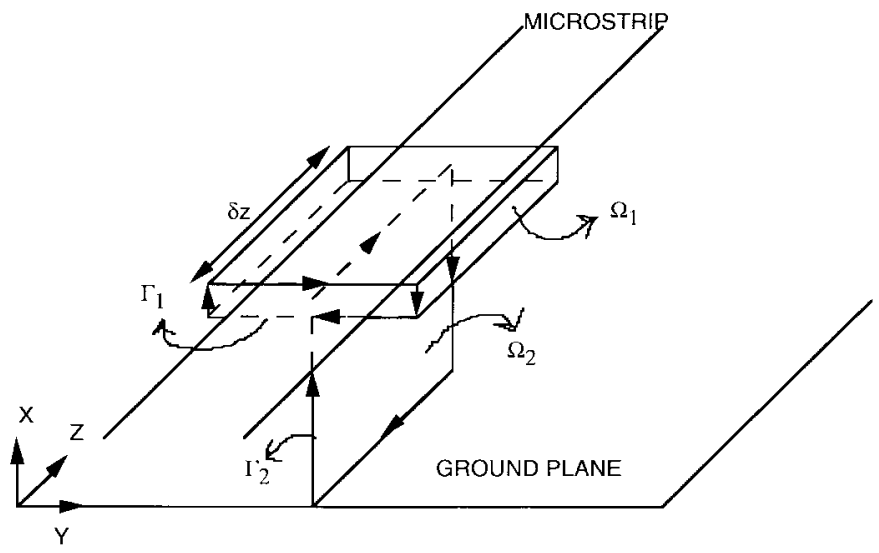

(b)

Fig. 1. (a) Schematics of the microstrip line with linewidth of $W$ and thickness of $h$, and (b) the closed Gaussian box $\Omega_{1}$ for the charge integration, the open surface $\Omega_{2}$ for the magnetic flux integration, the close loop $\Gamma_{1}$ for the current integration, and the line $\Gamma_{2}$ for the voltage integration.

where

$\delta z \quad$ unit length in the propagation direction;

$Q \quad$ charge enclosed by a Gaussian surface;

$V$ potential difference between the microstrip conductor and the ground plane;

$\psi \quad$ magnetic flux cutting through an open surface between the ground plane and the center of the microstrip line;

$I$ current on the microstrip conductor enclosed by a loop.

Referring to the schematics shown in Fig. 1, the $Q, V, \psi$, and $I$ are calculated by

$$
\begin{aligned}
Q & =\oint_{\Omega_{1}} \varepsilon \vec{E} \cdot d \vec{a} ; \quad \psi=\int_{\Omega_{2}} \mu \vec{H} \cdot d \vec{a} \\
I & =\oint_{\Gamma_{1}} \vec{H} \cdot d \vec{l} ; \quad V=\int_{\Gamma_{2}} \vec{E} \cdot d \vec{l} .
\end{aligned}
$$

The line integration in numerical form can be easily implemented in the FDTD method. The Fourier transformations are then performed on the time-dependent $Q, V, \psi$, and $I$ to obtain the frequency components for each quantity. The $C$ and $L$ are calculated as functions of frequency by $C(f)=Q(f) / V(f)$ and $L(f)=\psi(f) / I(f)$. The quantity of $L C$ is shown to be

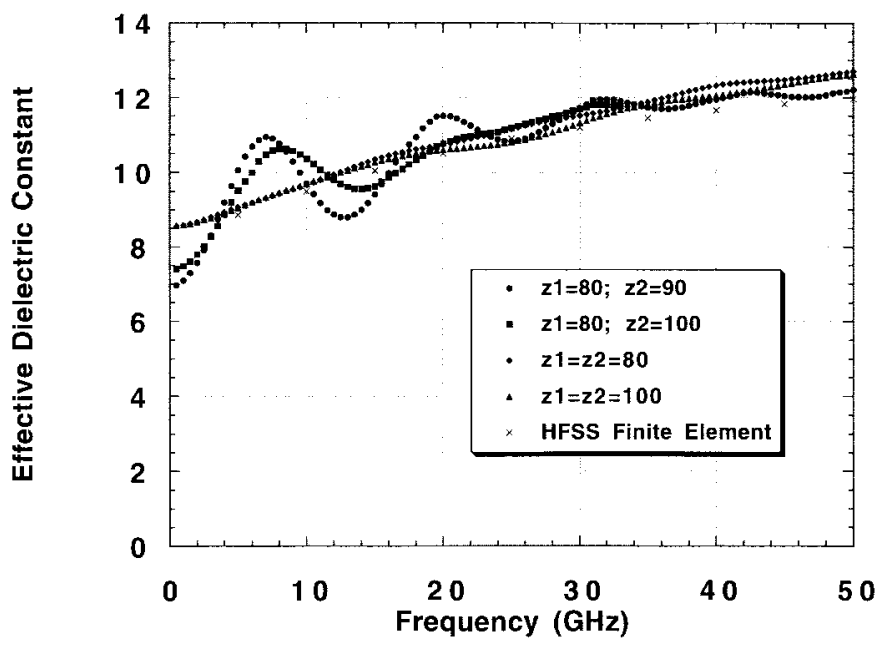

Fig. 2. The effective dielectric constant as a function of frequency.

the inverse of the square of the phase velocity in the following:

$$
\begin{aligned}
L C=\frac{\psi Q}{\Gamma V(\delta z)^{2}}=\frac{1}{\omega^{2}} & \frac{[V(z)-V(z+\delta z)]}{V(z) \delta z} \\
& \frac{[-I(z)+I(z+\delta z)]}{I(z) \delta z}=\frac{1}{v_{p}^{2}} .
\end{aligned}
$$

Therefore, the effective dielectric constant is given by

$$
\varepsilon_{\mathrm{eff}}=c^{2} L C
$$

and the characteristic impedance is given by

$$
Z_{c}=(L / C)^{1 / 2} \text {. }
$$

The similarity between these results and those derived using a transmission line model is expected. One notes, however, that as the frequency increases, the quasi-TEM property of the wave in the microstrip waveguide gradually deviates from a TEM wave. As a consequence, $E$ and $H$ fields are no longer conservative (i.e., the $V$ and $I$ values depend on the choice of the line integration path). Generally speaking, the equivalent circuit approach is only an approximation. As the frequency becomes higher, the propagation of the microwaves becomes more difficult to describe using linear circuit theory.

\section{NUMERICAL RESUlTS AND Discussions}

In this study, the FDTD method is implemented with a dispersive boundary condition [4]. A simple source excitation scheme, as used in [9], is used in the present study. The microstrip line is on an GaAs substrate (dielectric constant $\varepsilon$ is 12.9) with a thickness of $1000 \mu \mathrm{m}$. The width of the microstrip line is also $1000 \mu \mathrm{m}$. The computational domain contains $N_{x} \times N_{y} \times N_{z}=51 \times 104 \times 120$, and the spacial interval is $100 \mu \mathrm{m}$. The source plane is located at $z=20$ units. Fig. 2 shows the effective dielectric constant as a function of frequency between 0 and $50 \mathrm{GHz}$. The two oscillatory results are obtained using the multiple- $z$ FDTD method with two different pairs of $z$ values. The result with a larger swing corresponds to the pair of $z$ values with a smaller distance. These results demonstrate the drawback of the conventional FDTD method using multiple- $z$ values. The required accuracy 


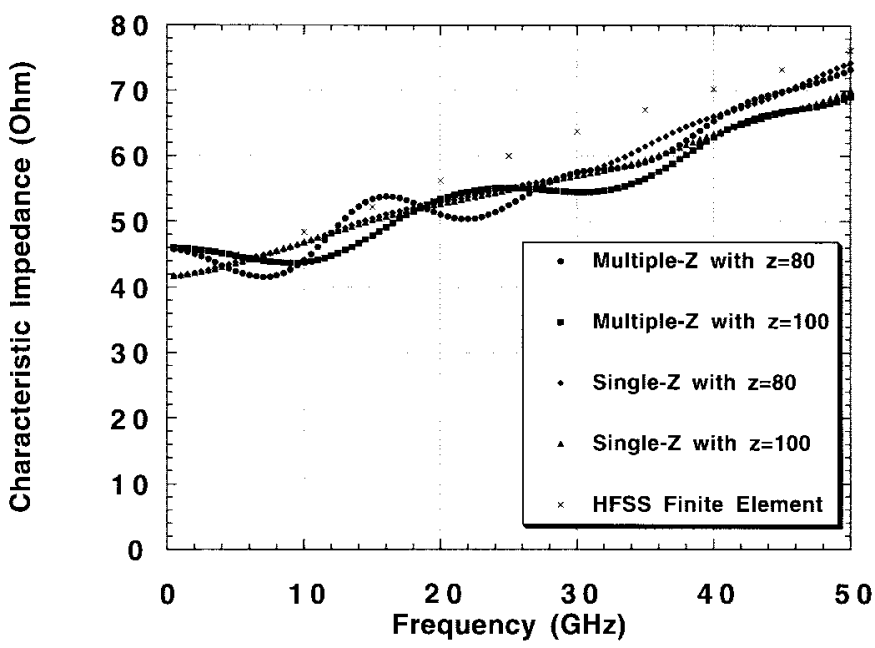

Fig. 3. The characteristic impedance as a function of frequency.

for a microwave design precludes the use of such results. The FDTD method using the equivalent circuit approach does not show oscillatory results. The values determined at different $z$-location is essentially unchanged until the frequency is higher than $20 \mathrm{GHz}$. Beyond $20 \mathrm{GHz}$, we suspect that the higher order modes are setting in and they are causing a slight difference in the effective dielectric constant evaluated at the different locations [6]. Fig. 3 shows the characteristic impedance as a function of frequency. Again, the traditional multiple- $z$ FDTD method shows oscillatory behavior, and different location of $z$ value gives different results. The FDTD method using the equivalent circuit approach shows consistent results regardless of the observation location in the direction of propagation. The computation using a high-frequency structure simulator (HFSS), a commercial software analysis tool based on finite-element frequency-domain calculation, was conducted. The dispersive effective dielectric constant of the default mode of the quasi-TEM mode was calculated. The characteristic impedance was calculated by dividing the voltage by the current. Their frequency dependence between 5 and $50 \mathrm{GHz}$ are also shown in Figs. 2 and 3. The results of the present approach agree well with the frequency-domain results for frequencies less than $20 \mathrm{GHz}$ where a quasi-TEM mode is a good assumption. Higher than $20 \mathrm{GHz}$, the quasiTEM mode further deviates from the TEM mode, and the numerical results depend on the definition for the voltage and the current. The paths taken in the line integrations for the voltage and current calculations depend on the grid structures, and they are different between the HFSS and the FDTD calculations. This may account for the slightly different results in that frequency region.

\section{CONCLUSION}

For microstrip line component analysis, we propose the use of an equivalent circuit approach using the FDTD method. In this approach, the capacitance and the inductance are evaluated at various monitor locations in the direction of propagation. Assuming the validity of the quasi-TEM approximation, the relations of the inductance, the capacitance, the phase velocity, and the characteristics impedance are derived. The results are shown to be the same as those for the traditional transmission line model. With the proposed approach, the phase velocity can be calculated based on the results of the FDTD analysis using a single- $z$ value. The traditional FDTD method to determine phase velocity is based on multiple- $z$ values. The latter method generates an oscillatory feature in the dispersion relations for the dielectric constant and characteristic impedance. This oscillatory behavior is minimized using the present approach with a single- $z$ method.

\section{REFERENCES}

[1] D. M. Sheen, S. M. Ali, M. D. Abouzahra, and J. A. Kong, "Application of the three-dimensional finite-difference time-domain method to the analysis of planar microstrip circuits," IEEE Trans. Microwave Theory Tech., vol. 38, pp. 849-856, July 1990.

[2] X. Zhang and K. K. Mei, "Time-domain finite difference approach to the calculation of the frequency-dependent characteristics of microstrip discontinuities," IEEE Trans. Microwave Theory Tech., vol. 36, pp. 1775-1787, Dec. 1988.

[3] X. Zhang, J. Fang, K. K. Mei, and Y. Liu, "Calculations of the dispersive characteristics of microstrips by the time-domain finite difference method," IEEE Trans. Microwave Theory Tech., vol. 36, pp. 263-267, Feb. 1988.

[4] Z. Bi, K. Wu, C. Wu, and J. Litva, "A dispersive boundary condition for microstrip component analysis using the FD-TD method," IEEE Trans. Microwave Theory Tech., vol. 40, pp. 774-777, Apr. 1992.

[5] J. Fang, "Absorbing boundary conditions applied to model wave propagation in microwave integrated-circuits," IEEE Trans. Microwave Theory Tech., vol. 42, pp. 1506-1513, Aug. 1994.

[6] Y. Wang and H. Ling, "Multimode parameter extraction for multiconductor transmission lines via single-pass FDTD and signal-processing techniques," IEEE Trans. Microwave Theory Tech., vol. 46, pp. 89-96, Jan. 1998.

[7] K. Naishadham and X. P. Lin, "Application of spectral domain Prony's method to the FDTD analysis of planar microstrip circuits," IEEE Trans. Microwave Theory Tech., vol. 42, pp. 2391-2398, Dec. 1994.

[8] A. Taflove, Computational Electrodynamics: The Finite-Difference Time-Domain Method. Norwood, MA: Artech House, 1995.

[9] A. P. Zhao, A. V. Raisanen, and S. R. Cvetkovic, "A fast and efficient FDTD algorithm for the analysis of planar microstrip discontinuities by using a simple source excitation scheme," IEEE Microwave Guided Wave Lett., vol. 5, pp. 341-343, Oct. 1995. 